\title{
Direct Characterization of the Spatial Effective Refractive Index Profile in Bragg Gratings
}

\author{
Zhaowei Zhang, Chun Tian, Michaël A. F. Roelens, Mohd R. Mokhtar, Periklis Petropoulos, David J. Richardson, \\ and Morten Ibsen
}

\begin{abstract}
We propose a pulse response method to directly characterize the phase of the spatial index modulation, including the dc refractive index distribution (distributed phase shift), discrete phase, and chirp of fiber Bragg gratings (FBGs). This method is based on the observation that the spatial phase of gratings is directly related to the temporal phase of its pulse response. Therefore, the phase of the spatial index modulation can be characterized directly by measuring the temporal phase of the pulse response of FBGs. This method is then used to characterize an FBG with a current-induced phase shift.
\end{abstract}

Index Terms-Fiber Bragg gratings (FBGs), impulse response, optical devices, spatial characterization.

\section{INTRODUCTION}

$\mathbf{F}$ IBER Bragg gratings (FBGs) with one or more phase shifts along their length are typically referred to as phase-shifted gratings. Phase shifts are usually introduced by imposing a spatial gap between two subgratings during fabrication. Phase shifts on FBGs can also be produced by applying an additional dc refractive index along the fiber [1]. Assuming that the distribution of the dc refractive index variation along the fiber is $\Delta n_{\mathrm{eff}}(x)$ from $x_{1}$ to $x_{2}$, the resultant phase shift can be represented by

$$
\phi=\frac{4 \pi}{\lambda_{B}} \int_{x_{1}}^{x_{2}} \Delta n_{\mathrm{eff}}(x) d x
$$

where $\lambda_{B}$ is the Bragg wavelength of the grating.

An example of how to achieve a distributed phase shift is shown in Fig. 1. A fine tungsten wire is put in contact with a uniform FBG and an electric current passes through the wire. The heat produced by this will affect the grating because the temperature increase causes an increase in the dc refractive index of the fiber, which consequently produces a phase shift in the grating. If the electrical current on the tungsten wire is tuned, different temperatures, and hence, different current-induced phase shifts, can be obtained.

This principle forms the basis of the optical code-division multiple-access (OCDMA) encoder-decoders, with multiple tunable phase shifts, that we have demonstrated in the past [1]. In their design and application, it is essential to know two features of the grating. One is the relationship between the

Manuscript received August 31, 2005; revised September 12, 2005.

Z. Zhang, C. Tian, M. A. F. Roelens, P. Petropoulos, D. J. Richardson, and M. Ibsen are with the Optoelectronics Research Centre, University of Southampton, Southampton SO17 1BJ, U.K. (e-mail: zhz@orc.soton.ac.uk).

M. R. Mokhtar was with the Optoelectronics Research Centre, University of Southampton, Southampton SO17 1BJ, U.K. He is now with Multimedia University, 63100 Cyberjaya, Selangor, Malaysia.

Digital Object Identifier 10.1109/LPT.2005.859527

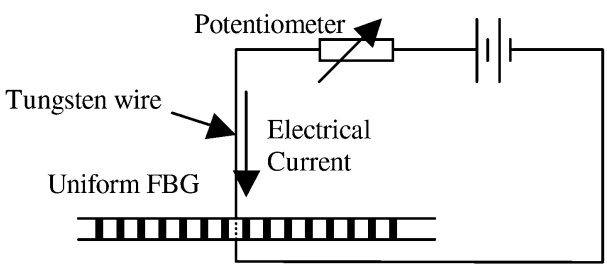

Fig. 1. Structure of an FBG with a wire-induced phase shift.

applied electrical current on the wire and the resultant phase shift, and the other is the spatial dc refractive index profile under the applied current.

Although various techniques, such as side-scattering [2], heat-scan [3], and optical low coherence reflectometry (OLCR) [4], have been proposed to measure the amplitude of spatial refractive index modulation of FBGs, little attention has been paid to the characterization of the phase of the spatial refractive index modulation. This includes the discrete phase shift, dc refractive index (or distributed phase shift), and chirp of FBGs. In [4], an OLCR technique, through which the impulse response data of the FBG can be achieved, was demonstrated to measure the spatial discrete phase shift and chirp of FBGs.

In this letter, we propose a method to characterize the dc refractive index profile of FBGs by directly measuring its pulse response. The spatial refractive index distribution of the grating is proved to be related to the temporal phase of its pulse response, and as a result, it can be directly retrieved from it. With this method, we experimentally characterize the tunable phase shifted FBG with different applied values of electrical currents to the thin tungsten wire.

\section{ChARACTERIZATION PRINCIPLE}

The characterization is based on measuring the pulse response of the FBG. A short optical pulse is reflected from the FBG under test. If the input pulse is short compared to the length of the grating, the reflected pulse response is a good approximation to its impulse response.

If the FBG under test is weak, the spatial phase of the grating will cause an equivalent temporal phase in its impulse response due to the space-time duality. Thus, the magnitude of the spatial phase shift of the grating can be approximately obtained from the magnitude of the temporal phase in its impulse response.

Furthermore, differentiation of (1) with respect to space $x$ gives

$$
\Delta n_{\mathrm{eff}}(x)=\frac{\lambda_{B}}{4 \pi} \frac{d \phi}{d x}
$$




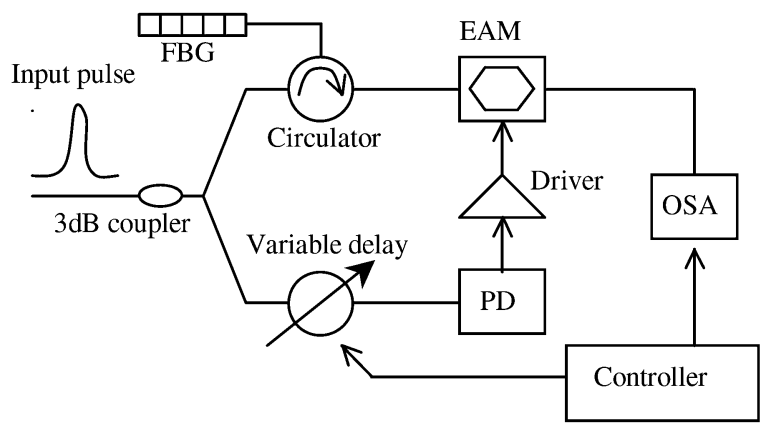

Fig. 2. Experimental setup.

Then, from the space-to-time conversion relationship [5]

$$
\Delta t=\frac{2 n_{\mathrm{eff}} \Delta x}{c}
$$

and the approximation that the temporal phase shift is equivalent to the spatial phase shift, a relationship between the dc refractive index distribution of the FBG and the temporal phase distribution of its impulse response can be obtained as

$$
\Delta n_{\mathrm{eff}}=\frac{\lambda_{B} \cdot n_{\mathrm{eff}}}{2 \pi c} \frac{\Delta \phi}{\Delta t} .
$$

Therefore, from (4), the spatial refractive index profile of an FBG can be determined by measuring the temporal phase distribution of its impulse response.

Based on the same principle, this method can also be used to characterize other grating parameters involving the phase of the spatial index modulation, such as the discrete phase shift and chirp in FBGs.

\section{EXPERIMENTS AND RESULTS}

To characterize an FBG with a tunable phase shift, we fabricated a $17-\mathrm{mm}$-long uniform grating in a standard single-mode fiber, and a $19-\mu \mathrm{m}$ diameter tungsten wire was placed in direct contact with the grating, $8 \mathrm{~mm}$ from one end, as shown in Fig. 1 . The Bragg wavelength, ac effective index modulation, peak reflectivity, and bandwidth of the FBG are, respectively, $1548 \mathrm{~nm}$, $3.3 \times 10^{-5}, 66 \%$, and $0.1 \mathrm{~nm}$.

The pulse response of the FBG is measured by the frequency-resolved optical-gating technique based on an electroabsorption modulator (EAM) [6]. The experimental setup is shown in Fig. 2. First, an input optical pulse train with 23 -ps pulses, at a repetition rate of $2.5 \mathrm{GHz}$, is split by a $3-\mathrm{dB}$ coupler. The central wavelength of the input pulse train is tuned to the Bragg wavelength of the FBG. In one arm, the pulses are reflected from the FBG under test, and then the reshaped optical pulses are incident into the EAM. In the other arm, the pulses pass through an optical stage with a controllable delay, and are detected by a fast photodiode and then amplified to generate a $2.5-\mathrm{GHz}$ sinusoidal electrical drive signal to the EAM as the switching window, which is synchronous with the optical pulse train incident to the EAM. The shaped optical pulses are, thus, optically sampled by the EAM. By varying the optical delay in a controlled fashion and measuring the optical spectra through an optical spectrum analyzer, (0.01-nm resolution, and $>70-\mathrm{dB}$ dynamic range), we obtain a spectrogram of the reflected optical pulses from the FBG. Then the electrical field
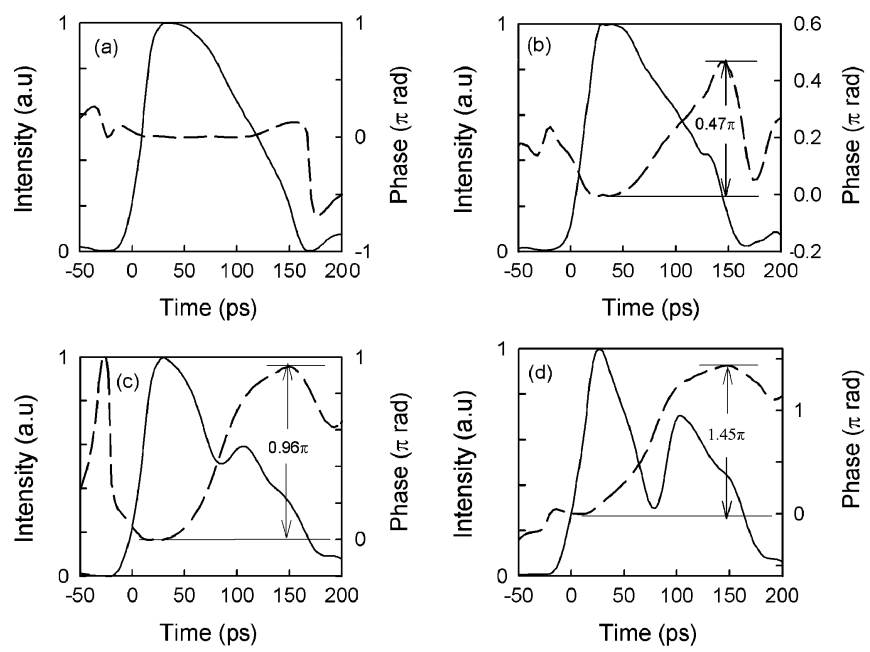

Fig. 3. Measured intensity (solid line) and phase (dashed line) of the reflected pulses from the FBG when the electrical currents along the tungsten wire are, respectively, (a) 0 , (b) 52, (c) 70, (d) $84 \mathrm{~mA}$.

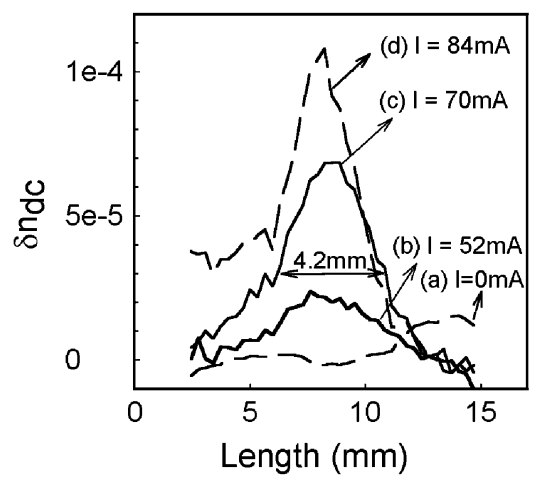

Fig. 4. Retrieved spatial refractive index distributions (a) when the current is $0 \mathrm{~mA}$. Retrieved spatial refractive index distributions induced by the electrical currents along the tungsten wire, when the currents are, respectively, (b) 52, (c) 70 , (d) $84 \mathrm{~mA}$.

(amplitude and phase) of the shaped pulses can be retrieved from its spectrogram [7].

The intensities and phases of the reflected pulses from the FBG are measured, and shown in Fig. 3, when the electrical currents applied on the tungsten wire are, respectively, 0, 52, 70, and $84 \mathrm{~mA}$. In Fig. 3, we can see that the temporal phase shifts are, respectively, $0,0.47 \pi, 0.96 \pi$, and $1.45 \pi$ at these different wire-currents. Therefore, we can deduce that the spatial phase shifts under these currents are approximately $0,0.47 \pi, 0.96 \pi$, and $1.45 \pi$.

The corresponding effective refractive index profiles under these electrical currents are calculated, using (4), and the results are plotted in Fig. 4. When the electrical current along the wire is $0 \mathrm{~mA}$, there is a small background refractive index profile on the uniform FBG. This might be from the nonuniformity in the fiber core [8]. When the electric current is $70 \mathrm{~mA}$, the full-width at half-modulation of the spatial refractive index distribution is $\sim 4.2 \mathrm{~mm}$.

\section{DISCUSSION}

The spatial resolution of the dc refractive index profile is limited by the input pulsewidth in the characterization. In this mea- 

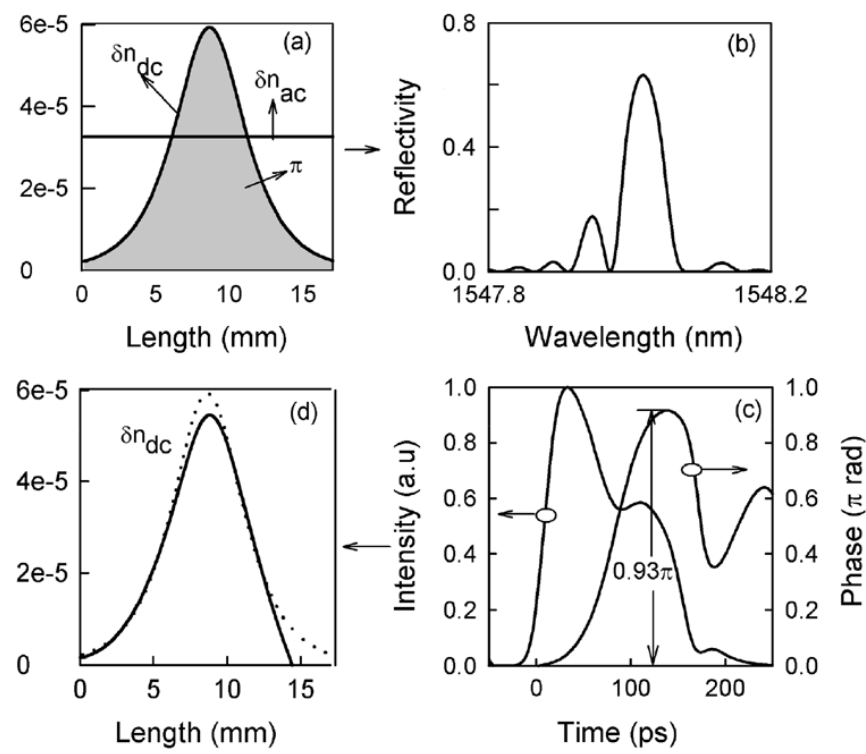

Fig. 5. (a) Assumed dc and ac refractive index distribution of an FBG with a $\pi$ phase shift, (b) its reflection spectrum, (c) intensity and phase of the reflected pulse from the grating (input pulsewidth $=23 \mathrm{ps}$ ), (d) retrieved (solid line) and assumed (dotted line) dc refractive index variation.

surement, the input pulsewidth is $23 \mathrm{ps,} \mathrm{so} \mathrm{the} \mathrm{spatial} \mathrm{resolution}$ is $\sim 2.3 \mathrm{~mm}$.

The strength of the grating will largely affect the measurement accuracy. As the strength of the grating increases, the equivalence of the amplitude of spatial phase shift and temporal phase shift will break gradually, so does the proportional relationship between the spatial refractive index distribution of the grating and the temporal phase differentiation of its pulse response.

A numerical simulation is shown in Fig. 5 to assess the accuracy of the experimentally retrieved results, which are affected by our practical choice of the input pulse and grating strength. In this simulation, we utilize the grating and input pulse having the same parameters as in the experiments. A 17-mm-long grating, with a uniform ac index modulation of $3.3 \times 10^{-5}$ and peak reflectivity of $66 \%$, has a spatial phase shift of $\pi$, consisting of a dc refractive index profile, as shown in Fig. 5(a), which is similar to the measured result of the $70-\mathrm{mA}$ wire-current case in the experiment. Then, a 23-ps optical pulse is reflected from the FBG. The temporal intensity and phase of the reflected pulse are calculated and shown in Fig. 5(c). The temporal phase is differentiated and then, using (4), the dc refractive index profile of the FBG is theoretically retrieved and is shown in the solid line of Fig. 5(d).

The deviation between the assumed and theoretically recovered results of phase shift and dc refractive index profile can be explained in the following argument. First, there is a deviation between the assumed spatial phase shift of $\pi$ and the retrieved temporal phase shift of $0.93 \pi$. This deviation is caused by the relatively high peak reflectivity, of $66 \%$, of the grating.
Second, as shown in Fig. 5(d), the spatial refractive index distribution is slightly smeared near its maximum, which is due to the spatial resolution limitation imposed by the input pulsewidth of 23 ps. Third, there is an obvious discrepancy of the refractive index profile at the rear part of the grating. This is caused by the high peak reflectivity of the grating, because less light can propagate to the rear part of the grating. This kind of simulation can give us a useful tool to analyze the accuracy of the practical experiments.

In the experiment, we directly measure the pulse response of the grating. Theoretically, it would be possible to obtain the accurate spatial information of the FBGs by deconvolution. Another possible approach is measuring its reflection spectrum (intensity and phase) response, and then retrieving the grating parameters through the inverse scattering technique [9]. But both of them are not practical, because they involve complex calculations, and the noise in the experimental data can affect the retrieved grating parameters seriously and unexpectedly [9].

\section{CONCLUSION}

We have proposed and experimentally demonstrated a pulse response method to directly measure the spatial phase shift and dc refractive index profile in FBGs. This method is based on the simple space-to-time conversion relationship. Its main advantage is that the dc refractive index distribution only locally depends on the temporal phase of the impulse response. The characterization results about the FBG, with a wire-current-induced phase shift, are fundamental in the design and analysis of tunable OCDMA encoder-decoders [1].

\section{REFERENCES}

[1] M. R. Mokhtar, M. Ibsen, P. C. Teh, and D. J. Richardson, "Reconfigurable multilevel phase-shift keying encoder-decoder for all-optical networks," IEEE Photon. Technol. Lett., vol. 15, no. 3, pp. 431-433, Mar. 2003.

[2] P. A. Krug, R. Stolte, and R. Ulrich, "Measurement of index modulation along an optical-fiber Bragg grating," Opt. Lett., vol. 20, pp. 1767-1769, 1995 .

[3] W. Margulis, I. C. S. Carvalho, and P. M. P. Gouvea, "Heat scan: A simple technique to study gratings in fibers," Opt. Lett., vol. 18, pp. 1016-1018, 1993.

[4] E. I. Petermann, J. Skaar, B. E. Sahlgren, R. A. H. Stubbe, and A. T. Friberg, "Characterization of fiber Bragg gratings by use of optical coherence-domain reflectometry," J. Lightw. Technol., vol. 17, no. 11, pp. 2371-2378, Nov. 1999.

[5] L. R. Chen, S. D. Benjamin, and P. W. E. Smith, "Ultrashort pulse reflection from fiber gratings: A numerical investigation," J. Lightw. Technol., vol. 15 , no. 8, pp. 1503-1512, Aug. 1997.

[6] C. Dorrer and I. Kang, "Real-time implementation of linear spectrograms for the characterization of high bit-rate optical pulse trains," IEEE Photon. Technol. Lett., vol. 16, no. 3, pp. 858-860, Mar. 2004.

[7] R. Trebino, K. W. DeLong, D. N. Fittinghoff, J. N. Sweetser, M. A. Krumbügel, and B. A. Richman, "Measuring ultrashort laser pulses in the time-frequency domain using frequency-resolved optical gating," Rev. Sci. Instrum., vol. 68, pp. 3277-3295, 1997.

[8] M. Ibsen and R. I. Laming, "Fiber nonuniformity caused Bragg grating imperfections," in OFC'99, San Diego, CA, Feb. 21-26, 1999, Paper FA1.

[9] J. Skaar and R. Feced, "Reconstruction of gratings from noisy reflection data," J. Opt. Soc. Amer. A, vol. 19, pp. 2229-2237, 2002. 